\title{
Thrombotic thrombocytopenic purpura or cobalamin deficiency? A case report and review
}

\author{
Neill Tuten ${ }^{1 *}$, Charles Bennett ${ }^{2}$ and William Babcock ${ }^{2}$ \\ ${ }^{1}$ Department of Medicine, University of South Carolina, USA \\ ${ }^{2}$ WJB Dorn VA Medical Center, Columbia, SC, USA
}

\begin{abstract}
Introduction: The classic presentation of cobalamin deficiency consists of a macrocytic anemia with or without neurologic manifestations. A common cause of cobalamin deficiency is pernicious anemia, an autoimmune condition in which anti-intrinsic factor antibodies are present. We present a case of pernicious anemia masked by features consistent with thrombotic thrombocytopenic purpura, as well as a review of similar cases in the literature.

Case presentation: A 31 year old male presented to the Emergency Department with symptomatic anemia and altered mental status. Laboratory data was significant for microangiopathic hemolytic anemia and thrombocytopenia. A clinical diagnosis of thrombotic thrombocytopenic purpura was made and plasma exchange was initiated. Due to the lack of improvement with plasma exchange, additional laboratory studies were obtained which showed cobalamin deficiency, intrinsic factor antibodies, and ADAMTS13 levels within normal limits. The patient's clinical and laboratory status improved with parenteral cobalamin replacement and discontinuation of plasma exchange.

Discussion: Severe cobalamin deficiency can present as a severe microangiopathic hemolytic anemia that responds rapidly to cobalamin replacement. Key differentiating considerations from thrombotic thrombocytopenic purpura are markedly elevated lactate dehydrogenase, low reticulocyte count, six-lobed polymorphonuclear white blood cells on the peripheral blood smear, and markedly low serum cobalamin.
\end{abstract}

\section{Introduction}

Classic presentation of vitamin $\mathrm{B}_{12}(\mathrm{Cbl})$ deficiency consists of a macrocytic anemia with or without neurologic manifestations. A common cause of Cbl deficiency is pernicious anemia (PA), an autoimmune condition in which anti-intrinsic factor (IF) antibodies are present.

Severe Cbl deficiency may be masked by features such as hemolytic anemia, thrombocytopenia, and schistocytosis, as outlined in a small number of case reports and small clinical series. Severe Cbl deficiency resulting in microangiopathic hemolytic anemia (MAHA) has been labeled in the literature as pseudo-thrombotic microangiopathy (pseudo-TMA). Misdiagnosis of Cbl deficiency as other conditions such as TTP may result in costly and unnecessary workups and treatments, such as prolonged plasma exchange.

Of 7 patients with $\mathrm{Cbl}$ deficiency and associated pseudo-TMA described by Noel et al. in 2013 [1], all had very high LDH levels and a low reticulocyte count, which should point toward a diagnosis of pseudo-TMA rather than TTP, as further described below.

The following is our case describing a patient with pseudo-TMA due to PA, mimicking TTP.

\section{Case presentation}

A 31 year old male with history of hypertension and hyperlipidemia, presented to the Emergency Department (ED) with complaints of shortness of breath, fatigue, and numbness and tingling of both hands.

Physical examination was notable for confusion, anxiety, restlessness, and an inability to fully cooperate with the complete neurologic examination.

Laboratory data was significant for a hemoglobin of $4.4 \mathrm{~g} / \mathrm{dL}, \mathrm{MCV}$ of $100.6 \mathrm{fL}$, platelet count of $87 \mathrm{~K} / \mathrm{cmm}$, total bilirubin of $2.7 \mathrm{mg} / \mathrm{dL}$, indirect bilirubin of $1.9 \mathrm{mg} / \mathrm{dL}, \mathrm{LDH}>665 \mathrm{U} / \mathrm{L}$, haptoglobin $<7.8 \mathrm{mg} /$ $\mathrm{dL}$, and normal coagulation studies. Schistocytes were present on the peripheral blood smear (Figures 1 and 2).

A working diagnosis of probable TTP was made. ADAMTS13 levels were ordered and plasma exchange was initiated promptly. The patient's clinical picture slightly improved with plasma exchange, but the thrombocyotopenia and schistocytosis persisted, and MCV and LDH levels remained elevated.

Further laboratory data showed Cbl of $68 \mathrm{pg} / \mathrm{mL}$, positive intrinsic factor antibodies, and rare six lobed polymorphonuclear blood cells were observed on the peripheral blood smear (Figures 3 and 4).

A revised diagnosis of PA was made. The patient's clinical and laboratory picture significantly improved after 2 doses of IM cyanocobalamin. Plasma exchange was discontinued after 6 sessions. The patient continued to improve clinically and serum platelet counts

Correspondence to: Neill Tuten, MD, Assistant Professor, Department of Medicine, 1 Medical Center Drive, PO Box 9156, Morgantown, WV 26506, West Virginia University, Tel: 803-300-2120; Fax: 304-293-2710; E-mail: neilltuten@gmail.com

Key words: pseudo-TMA, cobalamin (B12) deficiency, pernicious anemia

Received: July 15, 2015; Accepted: August 14, 2015; Published: August 17, 2015 


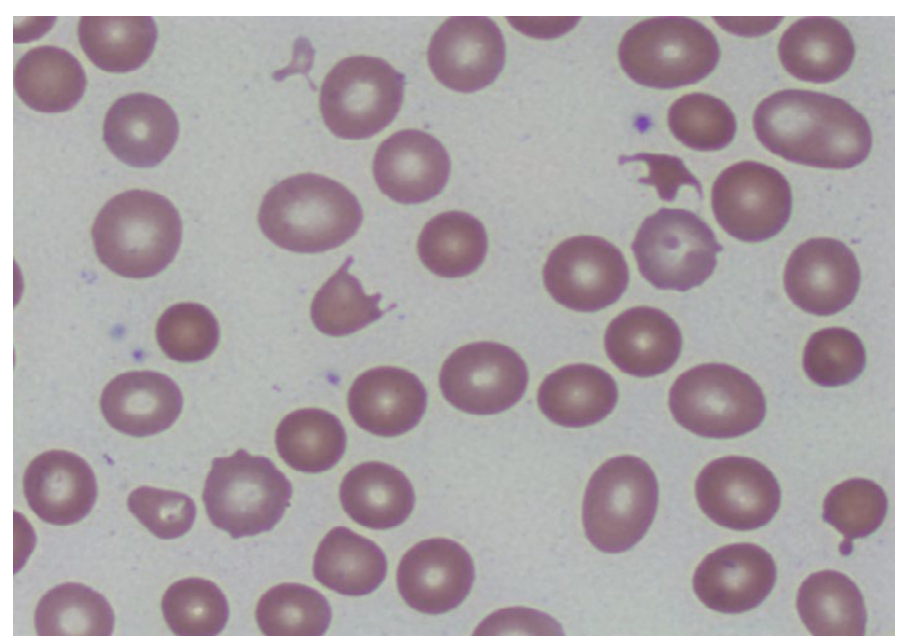

Figure 1. Peripheral smear showing schistocytes or helmet cells.

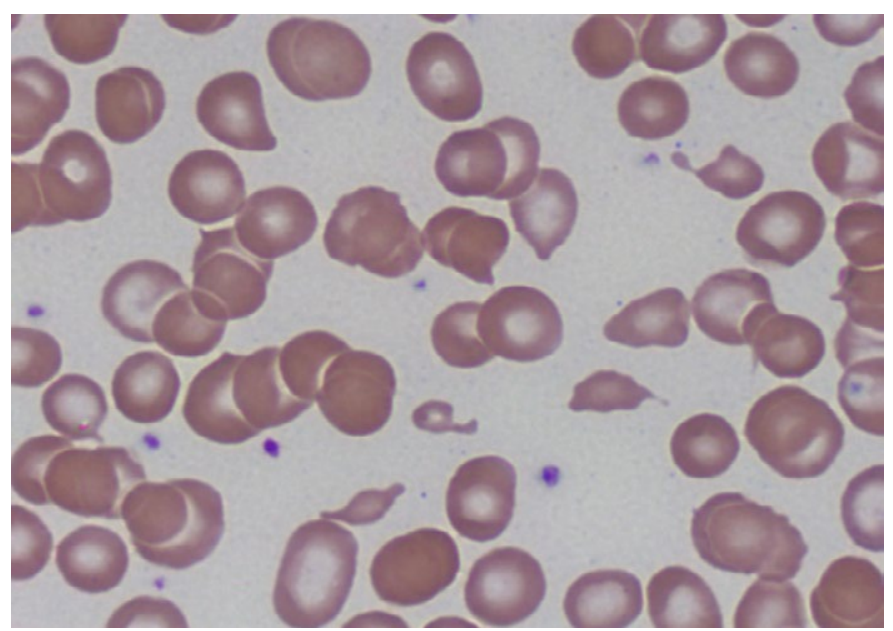

Figure 2. Peripheral smear showing schistocytes or helmet cells.

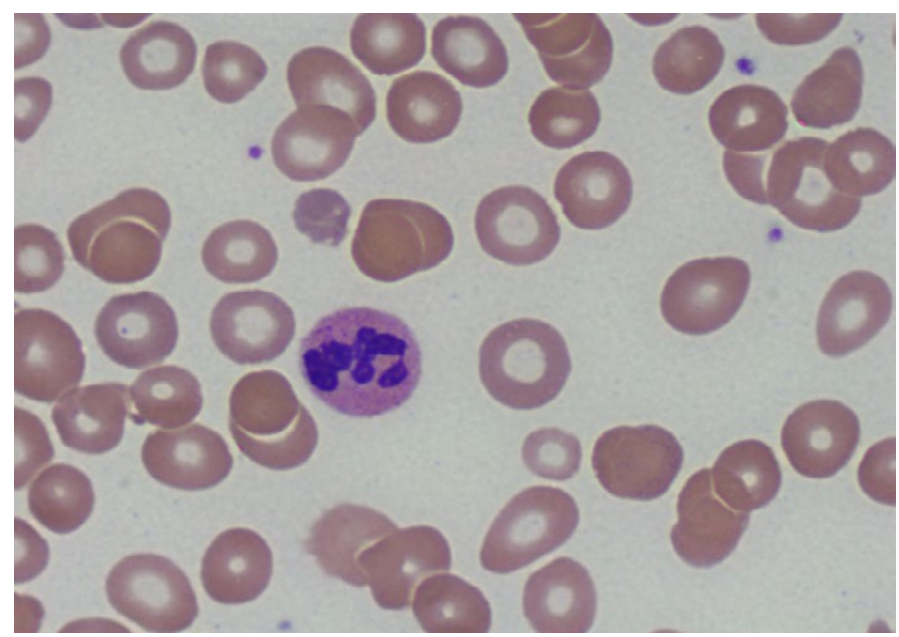

Figure 3. Peripheral smear showing hyper-segmented neutrophil.

rose with daily Cbl supplements. Subsequently, ADAMTS13 activity level was found not to be low.

Additional history obtained from family members indicated that the patient had had a cognitive deficit and personality issues for several

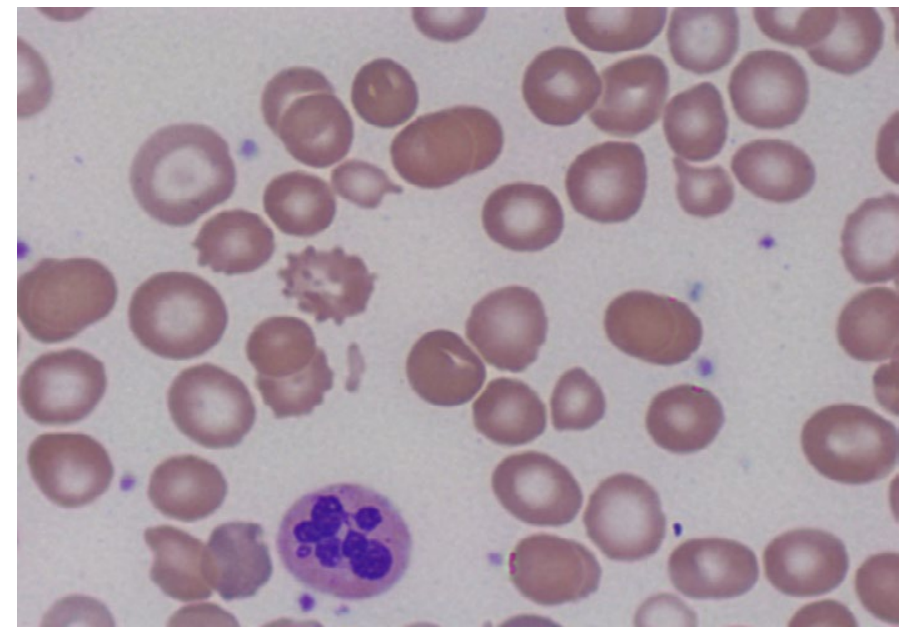

Figure 4. Peripheral smear showing hyper-segmented neutrophil.

years. Over a period of 3-4 days, decreased irritability and akathisias were noted, and all hematological abnormalities improved with continued Cbl replacement.

At follow-up appointment with a hematologist six weeks after discharge, the patient was noted to have continued altered mental ability although this had improved since hospitalization. Laboratory data were: hemoglobin $12.3 \mathrm{~g} / \mathrm{dL}$, hematocrit $38 \%$, MCV $76.6 \mathrm{fL}$, platelet count $189 \mathrm{~K} / \mathrm{cmm}$, LDH $192 \mathrm{U} / \mathrm{L}$, vitamin B12 $357 \mathrm{pg} / \mathrm{mL}$, reticulocyte count $1.28 \%$, total bilirubin $0.2 \mathrm{mg} / \mathrm{dL}$, and indirect bilirubin $0.1 \mathrm{mg} / \mathrm{dL}$.

At follow-up appointment 4 months after discharge, the patient's mental acuity remained unchanged. Laboratory data were: hemoglobin $14 \mathrm{~g} / \mathrm{dL}$, hematocrit 42.8\%, MCV $77.1 \mathrm{fL}$, platelet count $186 \mathrm{~K} / \mathrm{cmm}$, Cbl $669 \mathrm{pg} / \mathrm{mL}$.

Eight-month follow-up was notable for remarkable improvement in mental status, as evidenced by the patient's answering of questions more appropriately. Laboratory data at eight-month follow-up: hemoglobin $14.6 \mathrm{~g} / \mathrm{dL}$, hematocrit 44.4\%, MCV $83.2 \mathrm{fL}$, and platelet count $166 \mathrm{~K} / \mathrm{cmm}$.

\section{Discussion}

Red blood cell production is dependent on $\mathrm{Cbl}$. When $\mathrm{Cbl}$ is deficient, red blood cell membrane rigidity increases and erythrocyte deformability decreases [2]. These changes ultimately result in cell lysis, causing hemolytic anemia, and elevation of serum LDH levels [3]. LDH levels appear to be markedly higher among persons with MAHA due to Cbl deficiency versus TTP $[1,4]$. Cbl deficiency also causes elevated homocysteine levels, which leads to endothelial dysfunction and results in fragmentation of erythrocytes to schistocytes [5].

In 2011, Tadakamalla et al. described a patient with PA who was initially diagnosed with TTP based on hemolysis, thrombocytopenia and numerous schistocytes being identified in the initial peripheral blood smear. After four days of plasmapheresis, thrombocytopenia worsened. On re-evaluation, serum $\mathrm{Cbl}$ levels were low. The patient improved with intra-muscular Cbl treatment initiated after laboratory studies identified anti-IF antibodies and elevated serum gastrin levels, and an endoscopy identified atrophic gastritis [6].

In 2008, Dalsania et al. reported a patient who was also initially diagnosed with TTP based on severe thrombocytopenia that persisted 
following daily plasmapheresis. Additional evaluation led to a clinical diagnosis of pseudo-TMA secondary to PA [4]. Two similar cases of pseudo-TMA were reported previously in 2006 and 2003 [7,8].

In 2013, Noel et al. aimed to identify clinical and laboratory factors that could easily distinguish between TTP and pseudo-TMA related to Cbl deficiency. This retrospective study compared seven patients with pseudo-TMA and six patients with TTP. Pseudo-TMA patients had higher mean lactate dehydrogenase (LDH) levels (7310 vs. $1460 \mathrm{IU} / \mathrm{l}$, $\mathrm{P}=0.01)$, higher mean platelet counts ( 73 vs. $\left.12.5 \times 10^{9} / 1, \mathrm{P}=0.0023\right)$, lower mean reticulocyte count (13.1 vs. $\left.265.5 \times 10^{9} / 1, \mathrm{P}=0.0012\right)$ and lower mean neutrophil count $\left(1.3\right.$ vs. $\left.5.1 \times 10^{9} / \mathrm{l}, \mathrm{P}=0.0023\right)$ at presentation. They concluded that a very high LDH level and a low reticulocyte count should prompt screening for Cbl deficiency among persons who present with a clinical diagnosis of possible TTP [1].

Physicians should screen for Cbl deficiency when persons with a working diagnosis of TTP present with these laboratory findings. In contrast, acute renal failure and acute altered mental status point to TTP rather than pseudo-TMA. Our patient presented with a more chronic course of confusion and agitation rather than acute neurologic changes. Neurologic deficits persisted at six weeks after discharge, suggesting some degree of permanent neurological impairment due to long-standing $\mathrm{Cbl}$ deficiency. At eight month follow-up, our patient's mental status was remarkably improved.

Atypical presentations of common disorders can very easily be mistaken for other diseases, as in our case of pseudo-TMA secondary to $\mathrm{Cbl}$ deficiency mimicking TTP. Although severe Cbl deficiency is unusual, it still occurs. It is therefore prudent for clinicians to consider pseudo-TMA due to severe $\mathrm{Cbl}$ deficiency in the differential diagnosis for patients who present with a TTP-like picture with hemolytic anemia, thrombocytopenia, and schistocytosis.

We conclude that $\mathrm{Cbl}$ replacement may yield rapid clinical and hematological improvement among persons with severe Cbl deficiency who present with pseudo-TMA. Rapid diagnosis of pseudo-TMA due to severe $\mathrm{Cbl}$ deficiency and initiation of parenteral $\mathrm{Cbl}$ replacement may prevent unnecessary and expensive diagnostic workups and prolonged plasma exchange treatments.

\section{Competing interests}

The authors declare that they have no competing interests.

\section{Authors' contributions}

NT was the primary author composing, originally documenting the patients care as an inpatient and composing the case report. $\mathrm{CB}$ and $\mathrm{WB}$ are the attending hematologists who follow the patient; they contributed information regarding the progress of the patient after leaving hospital and contributed to the editing of the manuscript. All authors read and approved the final manuscript.

\section{Acknowledgements}

The authors would like to acknowledge the contributions of Dr. Nevene Andraws to this case. She is the pathologist who provided the peripheral smear images. She has no relevant financial disclosure.

\section{References}

1. Noël N, Maigné G, Tertian G, Anguel N, Monnet X, et al. (2013) Hemolysis and schistocytosis in the emergency department: consider pseudothrombotic microangiopathy related to vitamin B12 deficiency. QJM 106: 1017-1022. [Crossref]

2. Ballas SK, Saidi P, Constantino M (1976) Reduced erythrocytic deformability in megaloblastic anemia. Am J Clin Pathol 66: 953-957. [Crossref]

3. Aslinia F, Mazza JJ, Yale SH (2006) Megaloblastic anemia and other causes of macrocytosis. Clin Med Res 4: 236-241. [Crossref]

4. Dalsania CJ, Khemka V, Shum M, Devereux L, Lachant NA (2008) A sheep in wolf's clothing. Am J Med 121: 107-109. [Crossref]

5. Nappo F, De Rosa N, Marfella R, De Lucia D, Ingrosso D, et al. (1999) Impairment of endothelial functions by acute hyperhomocysteinemia and reversal by antioxidant vitamins. JAMA 281: 2113-2118. [Crossref]

6. Tadakamalla AK, Talluri SK, Besur S (2011) Pseudo-thrombotic thrombocytopenic purpura: A rare presentation of pernicious anemia. $N$ Am J Med Sci 3: 472-474. [Crossref]

7. Andrès E, Affenberger S, Federici L, Korganow AS (2006) Pseudo-thrombotic microangiopathy related to cobalamin deficiency. Am J Med 119: e3. [Crossref]

8. Garderet L, Maury E, Lagrange M, Najman A, Offenstadt G, et al. (2003) Schizocytosis in pernicious anemia mimicking thrombotic thrombocytopenic purpura. Am J Med 114: 423-425. [Crossref]

Copyright: (C2015 Tuten N. This is an open-access article distributed under the terms of the Creative Commons Attribution License, which permits unrestricted use, distribution, and reproduction in any medium, provided the original author and source are credited. 